\title{
CALAGEM, SILICATAGEM E DOSES DE FÓSFORO NO CRESCIMENTO E NUTRIÇÃO MINERAL DE ESTILOSANTES ${ }^{1}$
}

\author{
Jalison Lopes ${ }^{2}$, Antônio Ricardo Evangelista ${ }^{3}$, Caio Augustus Fortes ${ }^{3}$, \\ José Cardoso Pinto ${ }^{3}$, Antônio Eduardo Furtini Neto ${ }^{4}$, Ronan Magalhães de Souza ${ }^{5}$
}

\section{ABSTRACT \\ EFFECTS OF PHOSPHORUS RATES AND SOIL CORRECTIVES ON NUTRITIONAL EFFICIENCY OF Stylosanthes guianensis}

In tropical soils, soil acidity can increase the efficiency of phosphated fertilization. The objective of this study was to evaluate the effect of two soil correctives and phosphorus rates on nutritional efficiency of Stylosanthes guianensis cv. Mineirão. The experiment was carried out in a greenhouse at the Department of Soil Science, Universidade Federal de Lavras (UFLa), Minas Gerais State, Brazil. A randomized blocks design was used in a $5 \times 2$ factorial arrangement, with five $\mathrm{P}$ rates $\left(50 \mathrm{mg} \mathrm{dm}^{-3}, 100 \mathrm{mg} \mathrm{dm}^{-3}, 200 \mathrm{mg} \mathrm{dm}^{-3}, 400 \mathrm{mg} \mathrm{dm}^{-3}\right.$, and $800 \mathrm{mg} \mathrm{dm}^{-3}$ of soil) and two soil correctives (lime and $\mathrm{Ca}$ and $\mathrm{Mg}$ silicate), with three replicates. The use of silicate for soil correction provided higher accumulation of Mineirão dry matter rates, from $50 \mathrm{mg} \mathrm{dm}^{-3}$ to $420 \mathrm{mg} \mathrm{dm}^{-3}$ of $\mathrm{P}$. When phosphorus was associated with $\mathrm{Ca}$ and $\mathrm{Mg}$ silicate, the nutrients $\mathrm{P}, \mathrm{N}, \mathrm{Ca}$, $\mathrm{Mg}$, and $\mathrm{Zn}$ increased their use efficiency, resulting in a higher conversion rate of nutrients into biomass produced, except for the application of $800 \mathrm{mg} \mathrm{dm}^{3}$, due to nutritional imbalance in supplies of $\mathrm{P}$ and $\mathrm{Zn}$. The $\mathrm{Ca}$ and $\mathrm{Mg}$ silicate provided greater recovery of applied $\mathrm{P}$ and should be used for pasture formation and management where Mineirão is used.

KEY-WORDS: Stylosanthes guianensis cv. Mineirão; Ca and $\mathrm{Mg}$ silicate; lime; phosphorus utilization.

\section{INTRODUÇÃO}

A introdução de leguminosas forrageiras, nas pastagens formadas exclusivamente por gramíneas, é preconizada como alternativa de baixo custo, para aumento da qualidade e quantidade de forragem em oferta, além de permitir expressivas contribuições no fornecimento de nitrogênio $(\mathrm{N})$ ao sistema, via

\section{RESUMO}

Em solos tropicais, a correção da acidez do solo pode aumentar a eficiência da adubação fosfatada. Neste sentido, objetivou-se, por meio deste trabalho, avaliar o efeito de dois corretivos de acidez do solo e de doses crescentes de fósforo, sobre o crescimento e as eficiências nutricionais em Stylosanthes guianensis cv. Mineirão. O experimento foi conduzido em casa-de-vegetação do Departamento de Ciência do Solo (UFLa). O delineamento experimental utilizado foi o de blocos casualizados, com três repetições, com os tratamentos dispostos em esquema fatorial $5 \times 2$, constituídos por cinco doses de $\mathrm{P}\left(50 \mathrm{mg} \mathrm{dm}^{-3}, 100 \mathrm{mg} \mathrm{dm}^{-3}, 200 \mathrm{mg} \mathrm{dm}^{-3}\right.$, $400 \mathrm{mg} \mathrm{dm}^{-3}$ e $800 \mathrm{mg} \mathrm{dm}^{-3}$ de solo) e dois corretivos (calcário dolomítico e silicato de $\mathrm{Ca}$ e $\mathrm{Mg}$ ). A correção do solo com silicato proporcionou taxas de acúmulo de massa seca de estilosantes Mineirão mais elevadas, no intervalo de $50 \mathrm{mg} \mathrm{dm}^{-3}$ a $420 \mathrm{mg} \mathrm{dm}^{-3}$ de P. Os nutrientes $\mathrm{P}, \mathrm{N}, \mathrm{Ca}, \mathrm{Mg}$ e $\mathrm{Zn}$ tiveram suas eficiências de utilização aumentadas, quando a adubação fosfatada foi associada à silicatagem, ocorrendo maior conversão do nutriente em biomassa produzida, exceto para a dose $800 \mathrm{mg} \mathrm{dm}{ }^{3}$, em função do desbalanço nutricional nos fornecimentos de $\mathrm{P}$ e $\mathrm{Zn}$. A silicatagem proporcionou maior recuperação do $\mathrm{P}$ aplicado e deve ser usada na formação e manejo de pastagens onde o estilosantes Mineirão for utilizado.

PALAVRAS-CHAVE: Stylosanthes guianensis cv. Mineirão; silicato de $\mathrm{Ca}$ e $\mathrm{Mg}$; calcário; utilização do fósforo.

fixação biológica de N (FBN), pela associação leguminosa x Rhizobium.

$\mathrm{O}$ fósforo (P) é o nutriente que mais limita a produção de biomassa, em solos tropicais (Novais \& Smyth 1999). Os solos brasileiros apresentam alto potencial para adsorção do $\mathrm{P}$, reduzindo a disponibilidade para as plantas (Raij 1991, Novais \& Smyth 1999). Menos de 0,1\% do P encontra-se disponível em solução (Fardeau 1996).

1. Trabalho recebido em fev./2009 e aceito para publicação em jun./2010 (nº registro: PAT 5597/ DOI: 10.5216/pat.v40i2.5597).

2. Universidade Federal de Roraima, Escola Agrotécnica, Centro de Ciências Agrárias, Boa Vista, RR, Brasil.

E-mail: jalisonufla@gmail.com.

3. Universidade Federal de Lavras, Departamento de Zootecnia, Lavras, MG, Brasil. E-mails: aricardo@ufla.br, caioufla@yahoo.com.br, josecard@ufla.br.

4. Universidade Federal de Lavras, Departamento de Ciência do Solo, Lavras, MG, Brasil. E-mail: afurtini@ufla.br.

5. Centro Universitário de Patos de Minas, Faculdade de Engenharia e Ciências Agrárias, Departamento de Zootecnia, Patos de Minas, MG, Brasil.E-mail: ronan@unipam.edu.br. 
Em ensaios exploratórios de fertilidade do solo, constatou-se que o $\mathrm{P}$ foi o nutriente mais limitante ao crescimento de diversas leguminosas forrageiras tropicais, reduzindo, significativamente, os rendimentos de forragem e a nodulação e, consequentemente, os teores e quantidades acumuladas de $\mathrm{N}$ (Costa et al. 1989). No entanto, considerando-se o alto custo unitário dos fertilizantes fosfatados, tornase necessário assegurar sua máxima eficiência, por meio da determinação das doses mais adequadas ao estabelecimento e manutenção de pastagens. Além disto, técnicas como a correção da acidez do solo com silicatos podem aumentar a eficiência da adubação fosfatada, pelo fato de o ânion $\mathrm{H}_{3} \mathrm{SiO}_{4}^{-}$liberado por este corretivo concorrer pelo mesmo sítio de adsorção que o ânion fosfato $\left(\mathrm{H}_{2} \mathrm{PO}_{4}^{-}\right)$, contribuindo, assim, para reduzir seu grau de fixação pelo solo (Leite 1997).

Uma elevada disponibilidade de $\mathrm{P}$ pode induzir deficiência de $\mathrm{Zn}$, ocorrendo desordem no crescimento das plantas, a qual pode ser prevenida, ou corrigida, pelo fornecimento do micronutriente (Olsen 1972). A interação mais comum é encontrada quando a adição de $\mathrm{P}$ promove crescimento suficiente para diminuir a concentração de $\mathrm{Zn}$ nas plantas, ao nível de deficiência. Isto ocorre devido ao aumento da taxa de crescimento, pela aplicação de $\mathrm{P}$, mas sem que a taxa de absorção de $\mathrm{Zn}$ aumente de forma rápida o suficiente para manter a concentração necessária na parte aérea, promovendo diluição do $\mathrm{Zn}$ (Olsen 1972, Loneragan \& Weeb 1993).

A eficiência de uso de um nutriente pode ser definida como a relação entre a produção e a concentração do nutriente no tecido (Lauchli 1987), ou, ainda, como a quantidade de biomassa produzida por unidade do nutriente aplicado ao solo, sendo dependente de dois componentes principais: a eficiência de aquisição e a eficiência de utilização (Bailian et al. 1991). Para evitar a seleção de plantas eficientes na utilização de $\mathrm{P}$, porém com baixa produção, Siddiqi \& Glass (1981) propuseram o índice de eficiência: (matéria seca produzida) $2 /$ unidade do nutriente absorvido, reunindo, em um mesmo índice, a eficiência de utilização metabólica do nutriente e o crescimento.

Apesar de o Stylosanthes guianensis cv. Mineirão apresentar baixo a médio nível de exigência em P no solo, torna-se importante o conhecimento dos fatores nutricionais limitantes ao perfeito funcionamento da simbiose leguminosa-rizóbio, visando a maximizar a FBN, contribuindo, assim, para melhorar o estabelecimento e persistência desta espécie em pastagens.

Portanto, objetivou-se avaliar os efeitos de dois corretivos de acidez do solo (silicato de $\mathrm{Ca}$ e $\mathrm{Mg}$ ou calcário dolomítico) e de doses crescentes de fósforo, sobre o crescimento e as eficiências nutricionais em Stylosanthes guianensis cv. Mineirão.

\section{MATERIAL E MÉTODOS}

O experimento foi conduzido de agosto de 2006 a março de 2007, em casa-de-vegetação pertencente ao Departamento de Ciência do Solo da Universidade Federal de Lavras, Minas Gerais (UFLa). Foi utilizado solo classificado como Latossolo Vermelho-Escuro (LE), textura muito argilosa $(70 \%$ de argila), coletado em área de pastagem degradada do Departamento de Zootecnia da UFLa, na profundidade $0-20 \mathrm{~cm}$. Em seguida à coleta, o solo foi seco ao ar livre e peneirado em tamiz de $5 \mathrm{~mm}$. Após a secagem do solo, foram retiradas amostras destinadas à análise química e física (Tabela 1).

Após estes procedimentos iniciais e análises físicas e químicas, metade do solo foi submetido à calagem e a outra metade à silicatagem, levando-se em consideração o método de saturação por bases, para se elevar a saturação de base de $44 \%$ para $60 \%$, utilizando-se calcário dolomítico (99,02\% de PRNT, $36 \%$ de $\mathrm{CaO}$ e $9,0 \%$ de $\mathrm{MgO}$ ) e silicato de $\mathrm{Ca}$ e $\mathrm{Mg}$ (53\% de PRNT, $36 \%$ de $\mathrm{CaO}$ e $14 \%$ de $\mathrm{MgO}$ ). Decorrida a aplicação dos corretivos, o solo permaneceu incubado por 165 dias. Este período de incubação foi utilizado para garantir que a reação do silicato com o solo fosse completa, em virtude do baixo PRNT apresentado por este corretivo. Procurou-se evitar que

Tabela 1. Caracterização química do solo na camada 0-20 cm, antes da aplicação dos tratamentos (Lavras, MG, 2006).

\begin{tabular}{|c|c|c|c|c|c|c|c|c|c|c|c|c|c|}
\hline $\mathrm{pH}$ & $\mathrm{P}^{1}$ & K & $\mathrm{Ca}^{2+}$ & $\mathrm{Mg}^{2+}$ & $\mathrm{Al}^{3+}$ & $\mathrm{H}+\mathrm{Al}$ & SB & (t) & $(\mathrm{T})$ & \multirow[t]{2}{*}{ V } & $\mathrm{m}$ & $\mathrm{MO}$ & $\mathrm{P}^{*}$ \\
\hline $\mathrm{H}_{2} \mathrm{O}$ & \multicolumn{2}{|c|}{$\mathrm{mg} \mathrm{dm}^{-3}$} & \multicolumn{7}{|c|}{$\mathrm{cmolc} \mathrm{dm}^{-3}$} & & $\%$ & & $\mathrm{mg} \mathrm{L}^{-}$ \\
\hline 5,6 & 1,4 & 55 & 2,1 & 0,9 & 0,2 & 4,0 & 3,1 & 3,3 & 7,1 & 44 & 6 & 3,7 & 18,8 \\
\hline
\end{tabular}

* Extrator: Melich-1. 
a reação de neutralização com silicato continuasse a ocorrer durante a condução do experimento, o que poderia interferir nos resultados.

O solo foi acondicionado em vasos plásticos sem furos, com capacidade para $4,0 \mathrm{dm}^{3}$, que receberam as cinco doses de $\mathrm{P}\left(50 \mathrm{mg} \mathrm{dm}^{-3}, 100 \mathrm{mg} \mathrm{dm}^{-3}\right.$, $200 \mathrm{mg} \mathrm{dm}^{-3}, 400 \mathrm{mg} \mathrm{dm}^{-3}$ e $800 \mathrm{mg} \mathrm{dm}^{-3}$ de solo), na forma de $\mathrm{H}_{3} \mathrm{PO}_{4}$, e uma adubação básica de plantio, que constou de $120 \mathrm{mg} \mathrm{dm}^{-3} \mathrm{de} \mathrm{K}$, aplicado na forma de $\mathrm{K}_{2} \mathrm{SO}_{4}$, e dos micronutrientes $\mathrm{Zn}\left(1,59 \mathrm{mg} \mathrm{dm}^{-3}\right)$, B $\left(1,57 \mathrm{mg} \mathrm{dm}^{-3}\right), \mathrm{Cu}\left(0,33 \mathrm{mg} \mathrm{dm}^{-3}\right), \operatorname{Mn}\left(0,84 \mathrm{mg} \mathrm{dm}^{-3}\right)$, Mo $\left(0,15 \mathrm{mg} \mathrm{dm}^{-3}\right)$ e Co $\left(0,01 \mathrm{mg} \mathrm{dm}^{-3}\right)$, aplicados, respectivamente, nas formas de $\mathrm{ZnSO}_{4}, \mathrm{H}_{3} \mathrm{BO}_{3}$, $\mathrm{CuSO}_{4}, \mathrm{MnSO}_{4},\left(\mathrm{NH}_{4}\right)_{6}-\mathrm{Mo}_{7} \mathrm{O}_{24}$ e $\mathrm{CoCl}_{2}$. Após a aplicação dos nutrientes, foram retiradas amostras de solo de cada tratamento, para caracterizá-las pela análise química (Tabela 2).

A semeadura foi efetuada utilizando-se 20 sementes por vaso. O desbaste foi realizado 15 dias após a emergência, deixando-se cinco plantas por vaso. $\mathrm{O}$ delineamento experimental utilizado foi em blocos casualizados, com três repetições, sendo os tratamentos dispostos em esquema fatorial $5 \times 2$, constituídos por cinco doses de $\mathrm{P}\left(50 \mathrm{mg} \mathrm{dm}^{-3}\right.$, $100 \mathrm{mg} \mathrm{dm}^{-3}, 200 \mathrm{mg} \mathrm{dm}^{-3}, 400 \mathrm{mg} \mathrm{dm}^{-3}$ e $800 \mathrm{mg}$ $\mathrm{dm}^{-3}$ de solo) e dois corretivos (calcário dolomítico e silicato de cálcio e magnésio), totalizando 30 parcelas experimentais.
A umidade do solo foi ajustada para atingir $60 \%$ do volume total de poros e assim mantida, por meio de pesagens periódicas. As plantas foram conduzidas até 68 dias após semeadura, quando foi realizado o corte da sua parte aérea, a $5 \mathrm{~cm}$ do solo. Posteriormente, foi retirado o solo de cada vaso e as raízes separadas por lavagem, em água corrente, sobre peneira de $0,5 \mathrm{~mm}$ de malha. Todo o material coletado (parte aérea mais sistema radicular) foi seco em estufa com circulação de ar forçada, a $55^{\circ} \mathrm{C}$, por 72 horas, e, depois, pesado e triturado em moinho tipo Willey.

Em seguida, as amostras do material vegetal foram submetidas a análise química, segundo metodologia descrita por Silva (1998), para a determinação dos teores de N, e por Sarruge \& Haag (1974), para a determinação dos teores de $\mathrm{P}, \mathrm{Ca}, \mathrm{Mg}$ e Zn. A partir da matéria seca total produzida e do acúmulo do nutriente na planta, foram calculadas, para os nutrientes $\mathrm{P}, \mathrm{N}, \mathrm{Ca}, \mathrm{Mg}$ e Zn, a eficiência de utilização: $(E U)=(\text { matéria seca total produzida em gramas })^{2 /}$ (acúmulo total do nutriente na planta em miligramas) (Siddiqi \& Glass 1981); eficiência de absorção das raízes: $($ ERA $)=($ acúmulo total do nutriente na planta em miligramas)/(matéria seca de raízes em gramas) (Swiader et al. 1994); e eficiência de transporte ou translocação: $(\mathrm{ET})=$ (acúmulo do nutriente na parte aérea)/(acúmulo total do nutriente na planta) x 100

Tabela 2. Caracterização química do solo, após incubação e aplicação dos tratamentos (Lavras, MG, 2007).

\begin{tabular}{|c|c|c|c|c|c|c|c|c|c|c|}
\hline \multirow{4}{*}{ Atributos } & \multicolumn{10}{|c|}{ Corretivos } \\
\hline & \multicolumn{5}{|c|}{ Calcário dolomítico } & \multicolumn{5}{|c|}{ Silicato de $\mathrm{Ca}$ e $\mathrm{Mg}$} \\
\hline & \multicolumn{10}{|c|}{ Doses de $\mathrm{P}\left(\mathrm{mg} \mathrm{dm}^{-3}\right)$} \\
\hline & 50 & 100 & 200 & 400 & 800 & 50 & 100 & 200 & 400 & 800 \\
\hline pH em água & 6,1 & 6,3 & 6,4 & 6,3 & 6,2 & 6,4 & 6,2 & 6,3 & 5,9 & 5,9 \\
\hline P-Melich-1 (mg dm$\left.{ }^{-3}\right)$ & 11,9 & 22,3 & 45,9 & 132,4 & 247,5 & 18,9 & 22,9 & 63,4 & 148 & 249,6 \\
\hline $\mathrm{K}^{+}\left(\mathrm{mg} \mathrm{dm}^{-3}\right)$ & 175 & 187 & 168 & 200 & 172 & 218 & 218 & 212 & 200 & 184 \\
\hline $\mathrm{Ca}^{2+}\left(\mathrm{cmolc} \mathrm{dm}^{-3}\right)$ & 4,2 & 4,1 & 4,0 & 3,9 & 4,3 & 4,5 & 3,5 & 4,1 & 4,6 & 4,3 \\
\hline $\operatorname{Mg}^{2+}\left(\right.$ cmolc $\left.\mathrm{dm}^{-3}\right)$ & 1,5 & 1,4 & 1,4 & 1,4 & 1,6 & 1,3 & 1,0 & 1,3 & 1,4 & 1,5 \\
\hline $\mathrm{Al}^{3+}\left(\mathrm{cmolc} \mathrm{dm}^{-3}\right)$ & 0,0 & 0,0 & 0,0 & 0,0 & 0,0 & 0,0 & 0,0 & 0,0 & 0,0 & 0,0 \\
\hline $\mathrm{H}+\mathrm{Al}\left(\mathrm{cmolc} \mathrm{dm}^{-3}\right)$ & 2,1 & 2,1 & 2,3 & 2,6 & 2,9 & 2,1 & 2,9 & 2,3 & 2,6 & 3,2 \\
\hline $\mathrm{SB}\left(\mathrm{cmolc} \mathrm{dm}^{-3}\right)$ & 6,2 & 6,0 & 5,8 & 5,8 & 6,3 & 6,4 & 5,1 & 5,9 & 6,5 & 6,3 \\
\hline $\mathrm{t}\left(\mathrm{cmolc} \mathrm{dm}^{-3}\right)$ & 6,2 & 6,0 & 5,8 & 5,8 & 6,3 & 6,4 & 5,1 & 5,9 & 6,5 & 6,3 \\
\hline $\mathrm{T}\left(\mathrm{cmolc} \mathrm{dm}^{-3}\right)$ & 8,3 & 8,1 & 8,1 & 8,4 & 9,2 & 8,5 & 8,0 & 8,2 & 9,1 & 9,5 \\
\hline $\mathrm{V}(\%)$ & 74,5 & 74,0 & 71,7 & 69,1 & 68,6 & 75,2 & 63,6 & 72,1 & 71,5 & 66,2 \\
\hline $\mathrm{m}(\%)$ & 0,0 & 0,0 & 0,0 & 0,0 & 0,0 & 0,0 & 0,0 & 0,0 & 0,0 & 0,0 \\
\hline Matéria orgânica (\%) & 3,8 & 4,6 & 4,1 & 4,3 & 4,0 & 4,1 & 4,1 & 4,0 & 4,4 & 4,3 \\
\hline P-rem $\left(\mathrm{mg} \mathrm{L}^{-1}\right)$ & 11,2 & 11,2 & 11,2 & 11,8 & 11,8 & 10,9 & 13,6 & 12,2 & 11,5 & 15,6 \\
\hline $\mathrm{Si}\left(\mathrm{mg} \mathrm{dm}^{-3}\right)$ & 6,3 & 6,4 & 9,9 & 7,8 & 10,9 & 10,3 & 10,3 & 11,6 & 12,8 & 15,4 \\
\hline
\end{tabular}


(Li et al. 1991). Para o P, ainda foi calculada a eficiência de aproveitamento do $\mathrm{P}$ aplicado: $(\mathrm{EA}$ de $\mathrm{P})=$ (acúmulo de $\mathrm{P}$ na planta)/(quantidade de $\mathrm{P}$ aplicado) $\mathrm{x}$ 100 (Ramos 2007).

Os dados obtidos foram submetidos a análise de variância, com auxílio do programa Sisvar (Ferreira 2000). Os efeitos dos corretivos foram comparados pelo teste $\mathrm{F}$, ao nível de $5 \%$ de significância, e os efeitos das doses de $\mathrm{P}$ foram testados por meio de equações de regressão. Os modelos de regressão foram escolhidos com base no teste $\mathrm{F}$, com significância de $1 \%$ e $5 \%$ de probabilidade, e nos seus respectivos coeficientes de determinação. Para interação significativa a $5 \%$, pelo teste $\mathrm{F}$, procedeuse ao desdobramento das doses de $\mathrm{P}$, dentro de cada corretivo.

\section{RESULTADOS E DISCUSSÃO}

O acúmulo de matéria seca total foi influenciado pela interação doses de $\mathrm{P} x$ corretivos $(\mathrm{P}<0,05)$. Para o solo corrigido com silicato, o máximo acúmulo de matéria seca de estilosantes Mineirão (36,5 g/vaso) ocorreu com a dose estimada de $420 \mathrm{mg} \mathrm{dm}^{-3} \mathrm{e}$, posteriormente, reduziu-se, com os acréscimos nas doses de P. Para o solo corrigido com calcário, o acúmulo de matéria seca, em resposta aos incrementos nas doses de P, foi crescente, em todo o intervalo estudado (Figura 1).

No intervalo entre $50 \mathrm{mg} \mathrm{dm}^{-3}$ e $420 \mathrm{mg} \mathrm{dm}^{-3}$ de $\mathrm{P}$ aplicado, a taxa de aumento do acúmulo de MST do estilosantes Mineirão foi superior no solo corrigido com silicato, com posterior redução. É provável que, neste intervalo, a maior disponibilização de $\mathrm{Si}$,

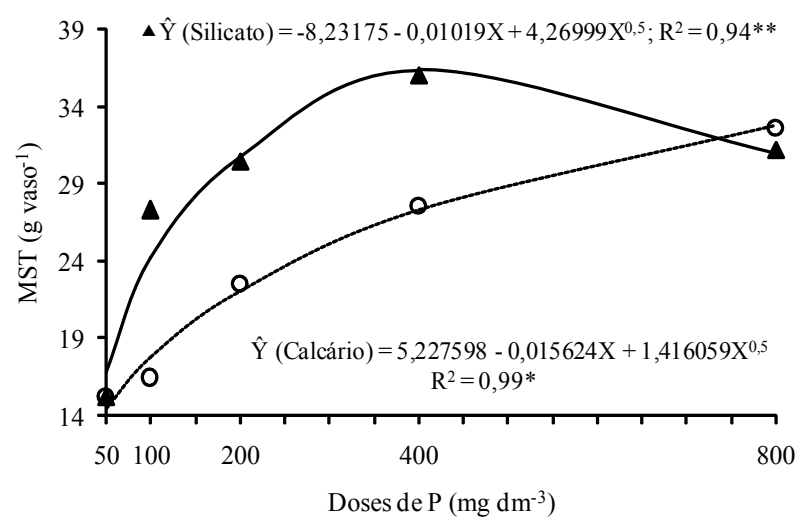

Figura 1. Acúmulo de matéria seca total (MST) do estilosantes Mineirão, em função de doses de P e corretivos (Lavras, MG, 2007). oriunda do corretivo, tenha favorecido a nodulação da leguminosa e, consequentemente, a FBN. Em trabalhos com feijão caupi, observou-se que a contagem e peso de nódulos foram influenciados positivamente, por doses crescentes de Si (Nelwamondo \& Dakora 1999).

Houve efeito da interação doses de $\mathrm{P} x$ corretivos sobre as eficiências de absorção de $\mathrm{P}(\mathrm{P}<0,01)$, $\mathrm{Ca}(\mathrm{P}<0,05), \mathrm{Mg}(\mathrm{P}<0,01)$ e $\mathrm{Zn}(\mathrm{P}<0,01)$. A eficiência de absorção de $\mathrm{N}$ foi influenciada apenas pelas doses de $\mathrm{P}(\mathrm{P}<0,01)$ (Figura 2).

No solo corrigido com calcário, a ERA de $\mathrm{P}$ aumentou, de forma linear, com os acréscimos nas doses de P. Para o solo corrigido com silicato, a ERA de $\mathrm{P}$, inicialmente, reduziu-se, chegando ao valor mínimo de $5,81 \mathrm{mg} \mathrm{g}^{-1}$, com a aplicação da dose estimada de $177 \mathrm{mg} \mathrm{dm}^{-3} \mathrm{de}$ P. Posteriormente, a ERA de $\mathrm{P}$ apresentou elevação acentuada, até a aplicação de $800 \mathrm{mg} \mathrm{dm}^{-3} \mathrm{de}$ P. Como o acúmulo de massa seca pelo estilosantes Mineirão foi mais favorecido, inicialmente, pela correção do solo com silicato, a ERA de P também reduziu-se, inicialmente. Como esta variável mede a razão entre o conteúdo do nutriente na planta e a produção de matéria seca de raízes, este comportamento ocorreu em função do efeito de diluição. A elevação na disponibilidade de $\mathrm{P}$ no solo (Tabela 2), combinada com a redução no acúmulo de massa seca do estilosantes Mineirão, a partir da aplicação de $420 \mathrm{mg} \mathrm{dm}^{-3} \mathrm{de} \mathrm{P}$, proporcionou aumento mais acentuado na ERA de $\mathrm{P}$, no solo corrigido com silicato. Isto se deve à maior concentração deste nutriente nos tecidos da leguminosa. Relaciona-se este comportamento a desbalanços no fornecimento de $\mathrm{P}$ e Zn, confirmados pela diagnose visual, para deficiência de $\mathrm{Zn}$ observada em plantas de estilosantes Mineirão, no solo corrigido com silicato e submetido às maiores doses de P. Observou-se, em algumas plantas, o surgimento de internódios curtos e folhas pequenas, dispostas em forma de roseta, com as folhas mais novas ficando menores e estreitas. Estes sintomas são semelhantes aos descritos por Oliveira et al. (2000), para a deficiência de Zn no capimtanzânia-1. Uma elevada disponibilidade de P pode induzir à deficiência de $\mathrm{Zn}$, ocorrendo desordem no crescimento das plantas (Olsen 1972).

Inicialmente, a absorção de $\mathrm{N}$ não aumentou na mesma proporção da massa radicular produzida, diluindo o $\mathrm{N}$ na planta e provocando redução na ERA de $\mathrm{N}$, tanto na presença do calcário como do silicato. Entretanto, a partir da aplicação de $100 \mathrm{mg} \mathrm{dm}^{-3}$ de $\mathrm{P}$ 

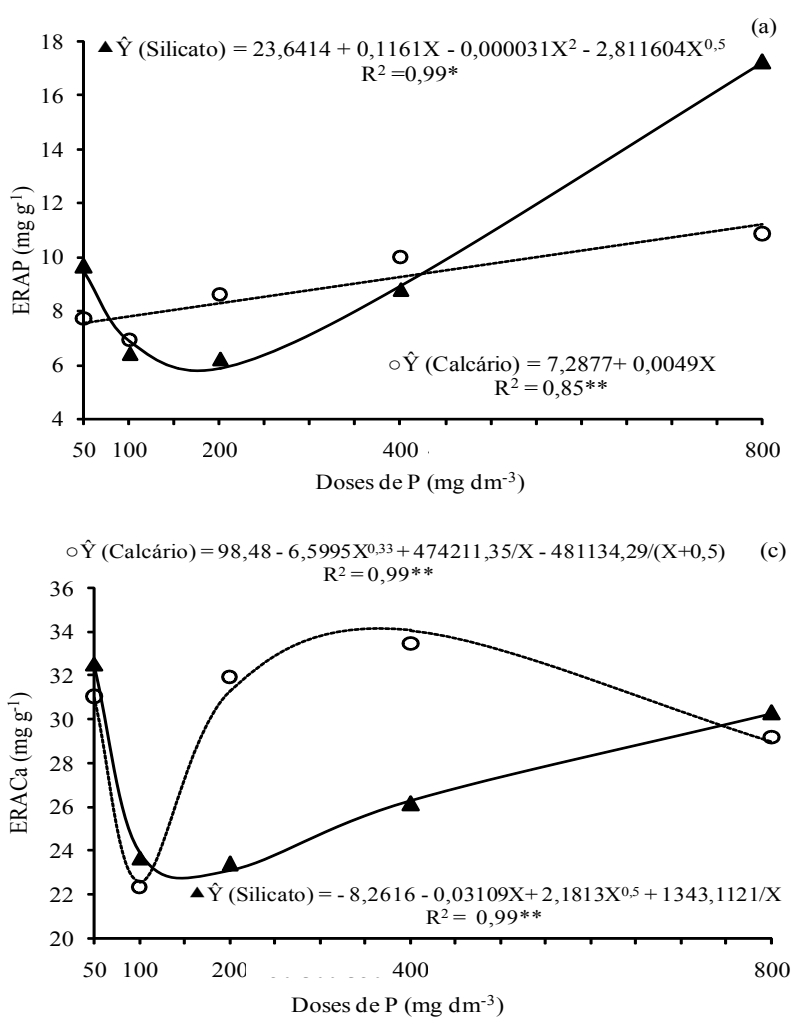

Figura 2. Eficiência das raízes do estilosantes Mineirão, na absorção de fósforo (a), nitrogênio (b), cálcio (c), magnésio (d) e zinco (e) (Lavras, MG, 2007).

associado ao silicato e $140 \mathrm{mg} \mathrm{dm}^{-3} \mathrm{de} \mathrm{P}$ associado ao calcário, volta a subir, pois o processo de infecção da raiz, por bactérias do gênero Rhizobium, é beneficiado pelo maior desenvolvimento radicular. Como a fixação biológica de $\mathrm{N}$ (FBN) é um processo bioquímico que necessita de energia na forma de ATP, o aumento na disponibilidade de P é capaz de favorecer este processo e, consequentemente, aumentar o conteúdo de $\mathrm{N}$ nos tecidos da planta. A FBN foi a principal via de fornecimento de $\mathrm{N}$, no presente estudo, já que nenhuma fonte mineral foi utilizada.

Os maiores valores de ERA de $\mathrm{Ca}$ e $\mathrm{Mg}$, observados na presença de calcário, se devem ao menor crescimento radicular, proporcional à taxa de absorção, aliado à baixa necessidade destes nutrientes pelo estilosantes Mineirão, comprovada pela redução na ERA de Ca e Mg, a partir da aplicação de $400 \mathrm{mg} \mathrm{dm}^{-3}$ de P. A partir desta dose de $\mathrm{P}$, o desenvolvimento radicular foi proporcionalmente mais acentuado que a taxa de absorção e a ERA de Ca e Mg reduziram-se.

Analisando-se o efeito das doses de P, com o uso do silicato como corretivo, verificou-se uma redução mais drástica da ERA de $\mathrm{Ca}$ e $\mathrm{Mg}$. Isto se
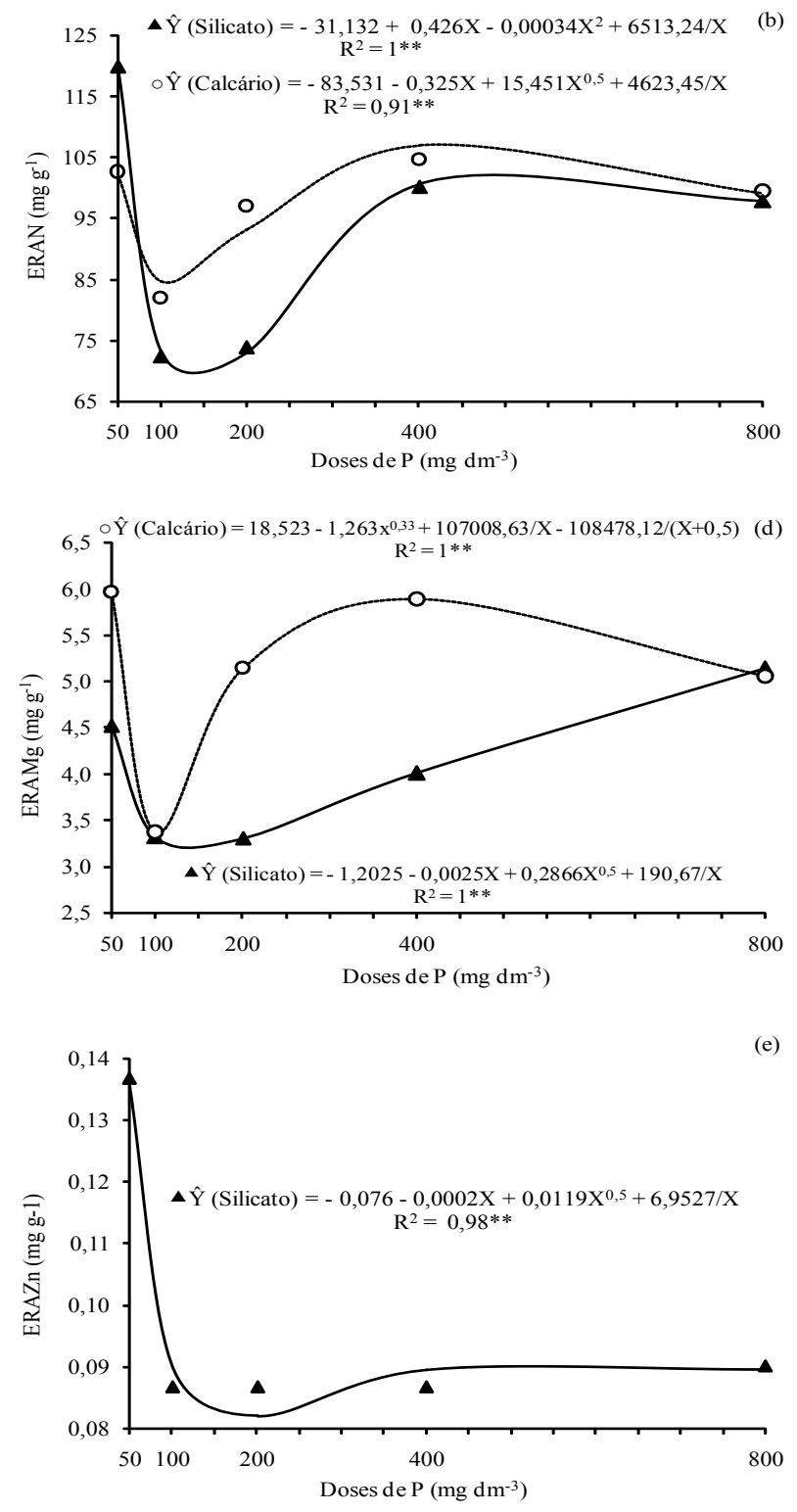

deve ao fato de esta leguminosa ser capaz de se desenvolver bem, com teores relativamente baixos de $\mathrm{Ca}$ e $\mathrm{Mg}$ em seus tecidos (Carvalho et al. 1988). Entretanto, o aumento da ERA de $\mathrm{Ca}$ e $\mathrm{Mg}$, observado a partir da aplicação de $200 \mathrm{mg} \mathrm{dm}^{-3}$, resultam de um aumento da necessidade da planta por estes nutrientes, para atender seus processos metabólicos, relacionados, principalmente, à produção de nódulos (Vitti et al. 2006).

A ERA de Zn, na presença de calcário, não se ajustou ao estudo de regressão, apresentando valor médio de $0,07 \mathrm{mg} \mathrm{g}^{-1}$ e mostrando-se pouco dependente das doses de P aplicadas. Entretanto, a ERA de Zn, na presença de silicato, reduziu-se drasticamente, no intervalo de $50 \mathrm{mg} \mathrm{dm}^{-3}$ a $100 \mathrm{mg} \mathrm{dm}^{-3}$ 
de $\mathrm{P}$, mantendo-se baixa para as demais doses aplicadas. $\mathrm{O}$ fato de a ERA de $\mathrm{Zn}$ não ter aumentando na presença de silicato, diferentemente do que ocorreu com a ERA de $\mathrm{P}, \mathrm{Ca}$ e $\mathrm{Mg}$, é indicativo de que o fator responsável pelo menor crescimento radicular, observado para este tratamento, foi a reduzida absorção de $\mathrm{Zn}$, ocasionada pela elevada disponibilidade de $\mathrm{P}$.

Houve efeito da interação doses de $\mathrm{P} x$ corretivos sobre as eficiências de utilização de $\mathrm{P}(\mathrm{P}<0,01)$, $\mathrm{N}(\mathrm{P}<0,05), \mathrm{Ca}(\mathrm{P}<0,01), \mathrm{Mg}(\mathrm{P}<0,01)$ e $\mathrm{Zn}$ $(\mathrm{P}<0,01)$ (Figura 3).

A correção do solo com silicato proporcionou aumento acentuado na EU de P, atingindo valor máximo de $3,8 \mathrm{~g}^{2} \mathrm{mg}^{-1}$, com a aplicação de $200 \mathrm{mg} \mathrm{dm}^{-3} \mathrm{de}$ $\mathrm{P}$, e posterior redução, com os acréscimos nas doses de P. Para o solo corrigido com calcário, a EU de P aumentou, no intervalo entre $50 \mathrm{mg} \mathrm{dm}^{-3} \mathrm{e} 400 \mathrm{mg} \mathrm{dm}^{-3}$ de $\mathrm{P}$, atingindo valor de $2,9 \mathrm{~g}^{2} \mathrm{mg}^{-1}$ e estabilizando-se, posteriormente. Comparando-se as máximas EU de $\mathrm{P}$ obtidas, em ambos os corretivos, verifica-se que a maior EU de $\mathrm{P}$, na presença de silicato, foi $23,7 \%$ superior à maior EU de $\mathrm{P}$ na presença de calcário, com metade da dose de $\mathrm{P}$ aplicada.
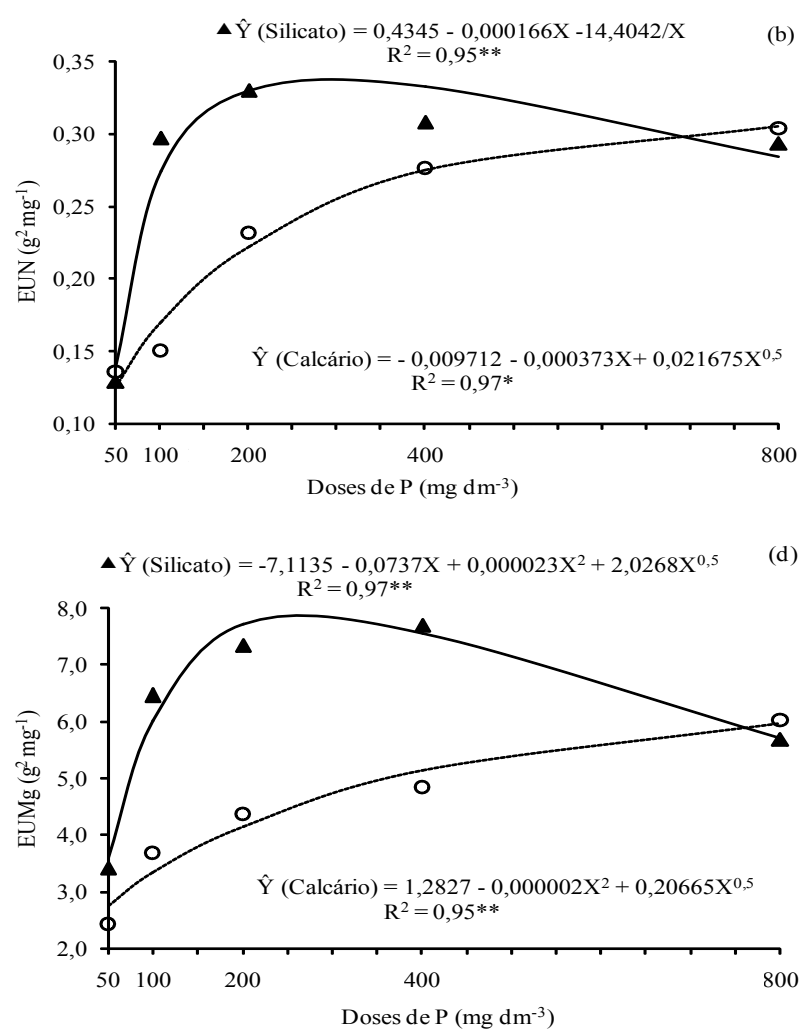

A redução na EU de $\mathrm{P}$, a partir da aplicação de $200 \mathrm{mg} \mathrm{dm}^{-3}$ de $\mathrm{P}$, na presença de silicato, se deve ao mais elevado teor de $\mathrm{P}$ na planta, em consequência da maior disponibilidade de $\mathrm{P}$ no solo, o que é resultado da menor conversão de $\mathrm{P}$ em biomassa, em razão dos desbalanços no fornecimento de $\mathrm{P}$ e Zn, já mencionados anteriormente. Vários fatores podem ser responsáveis pela ocorrência do efeito negativo que o $P$ é capaz de exercer sobre o Zn, prejudicando o crescimento da planta. Dentre estes fatores, destacase a combinação do $\mathrm{Zn}^{2+}$ com o $\mathrm{H}_{2} \mathrm{PO}_{4}^{2-}$, diminuindo a disponibilidade de ambos os nutrientes ( $\mathrm{Pe} \mathrm{Zn}$ ), em
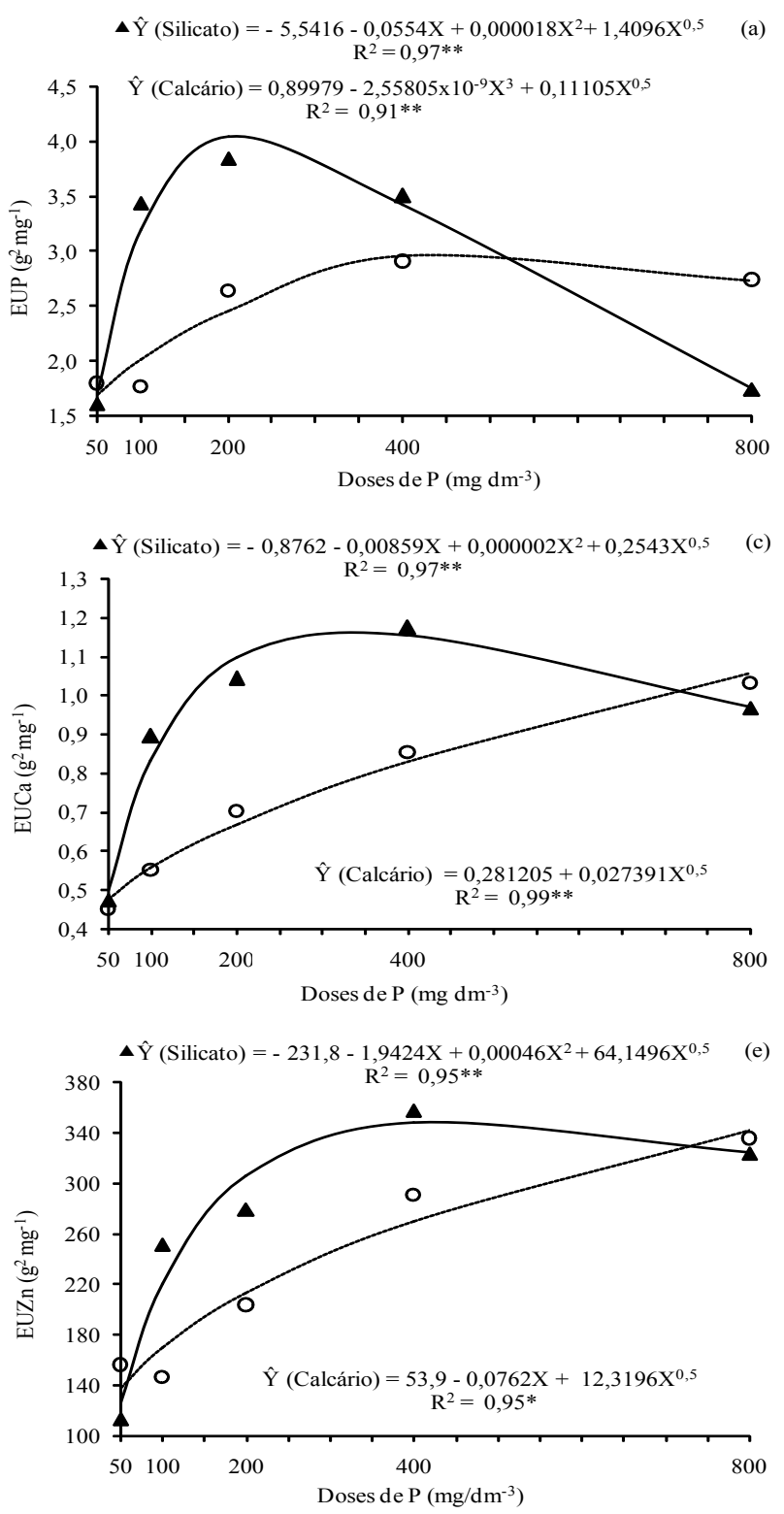

Figura 3. Eficiência do estilosantes Mineirão, na utilização de fósforo (a), nitrogênio (b), cálcio (c), magnésio (d) e zinco (e) (Lavras, MG, 2007). 
função da precipitação do fosfato de zinco formado; redução na translocação do $\mathrm{Zn}$ das raízes para a parte aérea, por causa do alto nível de fosfato nos tecidos condutores; e diminuição no teor de $\mathrm{Zn}$ da parte aérea, por um efeito de diluição, isto é, devido ao maior crescimento por efeito da fertilização fosfatada (Olsen 1972).

O Stylosanthes guianensis, apesar de apresentar uma boa capacidade de absorção de P, é pouco eficiente no uso deste nutriente, sobretudo com a aplicação de doses mais elevadas (Guss et al. 1990). Entretanto, no presente estudo, observou-se uma acentuada melhoria na utilização do $\mathrm{P}$, quando o silicato de $\mathrm{Ca}$ e $\mathrm{Mg}$ foram usados na correção do solo. Uma explicação provável para este efeito seria a presença do elemento Si no silicato, que é considerado benéfico para as culturas (Korndorfer et al. 2002, Faquin 2005). Neste sentido, a silicatagem, seguida da fertilização fosfatada, surge como estratégia para aumentar a eficiência de uso deste nutriente, mesmo para plantas com reconhecida capacidade de adaptação a solos de baixa fertilidade, como é o caso do estilosantes Mineirão.

A EU de $\mathrm{N}$ foi mais incrementada pelas doses de P na presença do silicato. Infere-se que a maior disponibilidade de $\mathrm{P}$, proporcionada pelo silicato, resultou em maior desenvolvimento radicular, aumentando os sítios de infecção na raiz, para serem colonizados pelas bactérias fixadoras de $\mathrm{N}$, além do maior fornecimento de $\mathrm{P}$, que é essencial para que a nodulação e FBN ocorram de forma satisfatória. Portanto, o aumento na EU de N, com o uso do silicato, pode ser relacionado à maior produção de massa radicular e maior nodulação, resultando em elevado ganho de matéria seca total produzida.

As EU de $\mathrm{Ca}, \mathrm{Mg}$ e $\mathrm{Zn}$, de maneira geral, comportaram-se de forma semelhante à EU de N, com os maiores valores sendo observados na presença do silicato. Isto demonstra que os benefícios observados para a EU de P, na presença do silicato, foram estendidos para os demais nutrientes utilizados. Usualmente, a eficiência de utilização diminui com o aumento da disponibilidade do nutriente no substrato (Silva et al. 1996). Nesta condição, a produção de biomassa pelas plantas não sofre incremento na mesma proporção que a absorção e acúmulo do nutriente nos tecidos, havendo, neste caso, declínio na utilização interna do nutriente, para a produção de biomassa (Siddiqi \& Glass 1981).
A eficiência de transporte do P absorvido (ET de P), ou eficiência de translocação, foi influenciada apenas pelos corretivos estudados $(\mathrm{P}<0,01)$, apresentando valor de 76,3\% para a leguminosa, na presença de calcário, contra $70,6 \%$, na presença de silicato, ou seja, aproximadamente 5,5\% maior para as plantas cultivadas no solo corrigido com calcário. A ET mede a capacidade da planta de transportar os nutrientes das raízes para a parte aérea e, comumente, plantas deficientes em $P$ retêm mais este nutriente nas raízes e o translocam menos para a parte aérea, em comparação com plantas bem supridas (Martinez et al. 1993). Entretanto, observou-se, neste estudo, que plantas bem supridas em $P$ também podem apresentar alta retenção de P no sistema radicular. Pode-se inferir que a maior retenção de $\mathrm{P}$ observada nas raízes, em função do uso do silicato como corretivo, não é devida a uma eventual carência de $\mathrm{P}$ disponível, mas sim a uma maior demanda energética deste órgão, para suplantar os processos de crescimento radicular e FBN mais intensificados. As eficiências de transporte do $\mathrm{N}, \mathrm{Ca}, \mathrm{Mg}$ e $\mathrm{Zn}$ não foram influenciadas por nenhum dos fatores em estudo $(\mathrm{P}>0,05)$, apresentando, em média, valores de $85,5 \% ; 89,5 \% ; 82,5 \%$; e $75,2 \%$, respectivamente. Assim, as diferentes doses de $\mathrm{P}$ aplicadas e os diferentes corretivos utilizados não exerceram efeito na distribuição destes nutrientes presentes na planta.

Houve efeito das doses de $\mathrm{P}$ sobre a EA de $\mathrm{P}$ pelo estilosantes Mineirão $(\mathrm{P}<0,01)$ (Figura 4).

A EA de $\mathrm{P}$ reduziu-se, com os aumentos nas doses de $\mathrm{P}$ aplicadas, variando de $13,2 \%$ para $3,2 \%$, da menor para a maior dose de $\mathrm{P}$ aplicada, respectivamente. A EA de $\mathrm{P}$ representa o valor percentual do $\mathrm{P}$

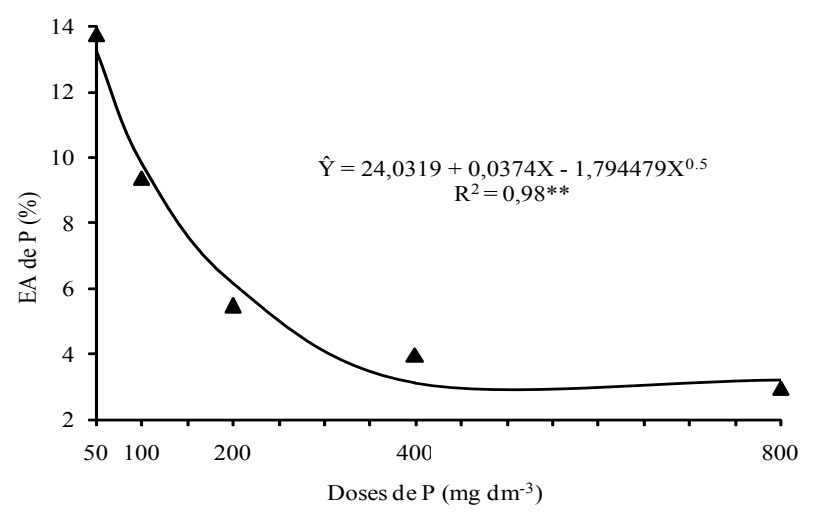

Figura 4. Eficiência de aproveitamento do fósforo aplicado pelo estilosantes Mineirão (Lavras, MG, 2007). 
aplicado ao solo, que foi absorvido e acumulado pela forrageira, e pode variar, dentre outros fatores, com a textura do solo, fontes de fertilizantes utilizadas e quantidade aplicada do nutriente. As leguminosas cultivadas no solo corrigido com silicato apresentaram maior EA de $\mathrm{P}(\mathrm{P}<0,01)$. O estilosantes Mineirão apresentou aproveitamento do $\mathrm{P}$ aplicado de $7,93 \%$, no solo corrigido com silicato, contra $6,24 \%$, no solo corrigido com calcário. Com base neste resultado, pode-se inferir que a silicatagem proporciona maior recuperação do $\mathrm{P}$ aplicado que a calagem. A maior liberação de $\mathrm{P}$, para a solução do solo, pela ação do ânion silicato $\left(\mathrm{H}_{3} \mathrm{SiO}_{4}^{-}\right)$, que concorre com o ânion fosfato $\left(\mathrm{H}_{2} \mathrm{PO}_{4}^{-}\right)$pelo mesmo sítio de adsorção, pode ter sido o principal fator responsável por este resultado. Este fenômeno assume importância ainda maior, em solos de textura muito argilosa, como o que foi usado no presente experimento, pois solos com estas características apresentam alta capacidade de fixação de $\mathrm{P}$. Aliada à maior disponibilização de $\mathrm{P}$, no solo onde se fez a silicatagem, a maior capacidade de exploração das raízes, em virtude de um maior desenvolvimento do sistema radicular, também pode ter contribuído sobremaneira para aumentar a aquisição de $\mathrm{P}$.

\section{CONCLUSÕES}

1. A adubação fosfatada sobre o estilosantes Mineirão, quando associada à silicatagem, aumenta a eficiência de utilização dos nutrientes $\mathrm{P}, \mathrm{N}, \mathrm{Ca}$, $\mathrm{Mg}$ e $\mathrm{Zn}$.

2. A silicatagem proporciona maior recuperação do P aplicado e deve ser usada para formação de pastagens de estilosantes Mineirão.

\section{REFERÊNCIAS}

BAILIAN, L.; McKEAND, S. E.; ALLEN, H. L. Genetic variation in nitrogen use efficiency of lablolly pine seedlings. Forest Science, London, v. 37, n. 2, p. 613628, 1991.

CARVALHO, M. M. et al. Respostas de leguminosas forrageiras tropicais à calagem e ao fósforo, em casade-vegetação. Revista Brasileira de Ciência do Solo, Campinas, v. 12, n. 2, p. 153-159, 1988.

COSTA, N. de L.; GONÇALVES, C. A.; OLIVEIRA, J. R. da C. Nutrientes limitantes ao crescimento de Brachiaria humidicola consorciada com leguminosas em Porto VelhoRO. Porto Velho: Embrapa-Uepae Porto Velho, 1989. (Comunicado técnico, 70).
FAQUIN, V. Nutrição mineral de plantas. Lavras: UFLa/ Faepe, 2005.

FARDEAU, J. C. Dynamics of phosphate in soils: an isotopic outlook. Fertility Research, Bonn, v. 45, n. 2, p. 91-100, 1996.

FERREIRA, D. F. Análises estatísticas por meio do Sisvar para Windows versão 4.0. In: REUNIÃO ANUAL DA REGIÃO BRASILEIRA DA SOCIEDADE INTERNACIONAL DE BIOMETRIA, 45., 2000, São Carlos. Anais... São Carlos: UFSCar, 2000. p. 255-258.

GUSS, A.; GOMIDE, J. A.; NOVAIS, R. F. Exigências de fósforo para estabelecimento de quatro leguminosas forrageiras em solos com distintas características físicoquímicas. Revista da Sociedade Brasileira de Zootecnia, Viçosa, v. 19, n. 5, p. 450-458, 1990.

KORNDORFER, G. H.; PEREIRA, H. S.; CAMARGO, M. S. de. Silicatos de cálcio e magnésio na agricultura. Uberlândia: UFU/ICIAG, 2002. (Boletim técnico, 1).

LAUCHLI, A. Soil science in the next twenty-five years: does a biotechnology play a role? Soil Science Society of America Journal, Madison, v. 51, n. 6, p. 1405-1409, 1987.

LEITE, P. C. Interação silício-fósforo em Latossolo Roxo cultivado com sorgo em casa-de-vegetação. 1997. $84 \mathrm{f}$. Tese (Doutorado em Agronomia)-Universidade Federal de Viçosa, Viçosa, 1997.

LI, B.; McKEAND, S. E.; ALLEN, H. L. Genetic variation in nitrogen use efficiency of loblolly pine seedlings. Forest Science, Bethesda, v. 37, n. 2, p. 613-626, 1991.

LONERAGAN, J. F.; WEBB, M. J. Interactions between zinc and other nutrients affecting the growth of plants. In: ROBSON, A. D. (Ed.). Zinc in soil and plants. Madison: Kluwer Academic, 1993. p. 119-134.

MARTINEZ, H. E. P. et al. Comportamento de variedades de soja cultivadas sob diferentes níveis de fósforo. II. Translocação do fósforo absorvido e eficiência nutricional. Revista Brasileira de Ciência do Solo, Campinas, v. 17, n. 2, p. 239-244, 1993.

NELWAMONDO, A.; DAKORA, F. D. Silicon promotes nodule formation and nodule function in symbiotic cowpea (Vigna unguiculata). New Phytology, Oxford, v. 142, n. 3, p. 463-467, 1999.

NOVAIS, R. F.; SMYTH, T. J. Fósforo em solo e planta em condições tropicais. Viçosa: UFV, 1999.

OLIVEIRA, I. P. et al. Efeitos qualitativo e quantitativo da aplicação do zinco no capim Tanzânia-1. Pesquisa Agropecuária Tropical, Goiânia, v. 20, n. 1, p. 43-48, 2000. 
OLSEN, S. R. Micronutrient interaction. In: MORTVEDT, J. J.; GIORDANO, P. M.; LINDSAY, W. L. (Eds.). Micronutrients in agriculture. Madison: Soil Science Society of America, 1972. p. 243-264.

RAIJ, B. van. Fertilidade do solo e adubação. Piracicaba: Ceres/Potafos, 1991.

RAMOS, J. Produção e utilização de fósforo por gramíneas forrageiras adubadas com fertilizantes fosfatados e pela soja e feijoeiro cultivados em sucessão. 2007. $63 \mathrm{f}$. Dissertação (Mestrado em Ciência do Solo)-Universidade Federal de Lavras, Lavras, 2007.

SARRUGE, J. R.; HAAG, H. P. Análises químicas em plantas. Piracicaba: ESALQ, 1974.

SIDDIQI, M.Y.; GLASS, A. D. M. Utilization index: a modified approach to the estimation and comparison of nutrient utilization efficiency in plants. Journal of Plant Nutrition, Monticello, v. 4, n. 3, p. 289-302, 1981.
SWIADER, J. M.; CHYAN, Y.; FREIJI, F. G. Genotypic differences in nitrate uptake and utilization efficiency in pumpkin hybrids. Journal of Plant Nutrition, Monticello, v. 17, n. 10 , p. 1687-1699, 1994.

SILVA, D. J. Análise de alimentos: métodos químicos e biológicos. Viçosa: UFV, 1998.

SILVA, I. R.; FURTINI NETO, A. E.; VALE, F. R. Eficiência nutricional para potássio em espécies florestais nativas. Revista Brasileira de Ciência do Solo, Campinas, v. 20, n. 2, p. 257-264, maio/ago. 1996.

VITTI, G. C.; LIMA, E.; CICARONE, F. Cálcio, magnésio e enxofre. In: FERNANDES, M. S. (Ed.). Nutrição mineral de plantas. Viçosa: Sociedade Brasileira de Ciência do Solo, 2006. p. 299-326. 